UNIVERSIDADE DE SÃO PAULO - FEUSP

CRISTINA MARIA COIN DE CARVALHO

OS PROFESSORES FRENTE ÀS DIFERENÇAS: UM ESTUDO DE SEU PATRIMÔNIO PEDAGÓGICO NO CICLO II DE UMA ESCOLA PÚBLICA 


\section{OS PROFESSORES FRENTE ÀS DIFERENÇAS: UM ESTUDO DE SEU PATRIMÔNIO PEDAGÓGICO NO CICLO II DE UMA ESCOLA PÚBLICA \\ Versão corrigida}

Dissertação de Mestrado apresentada ao Programa de Pós-Graduação da Faculdade de Educação da Universidade de São Paulo para obtenção do título de Mestre em Educação.

Área de concentração: Didática, Teorias de Ensino e Práticas Escolares.

Orientadora: Prof ${ }^{a}$ Dra. Vivian Batista da Silva 
AUTORIZO A REPRODUÇÃO E DIVULGAÇÃO TOTAL OU PARCIAL DESTE TRABALHO, POR QUALQUER MEIO CONVENCIONAL OU ELETRÔNICO, PARA FINS DE ESTUDO E PESQUISA, DESDE QUE CITADA A FONTE.

Catalogação na Publicação

Serviço de Biblioteca e Documentação

Faculdade de Educação da Universidade de São Paulo

371.9

Carvalho, Cristina Maria Coin de

C331p

Os professores frente às diferenças: um estudo de seu patrimônio pedagógico no ciclo II de uma escola pública / Cristina Maria Coin de Carvalho; orientação Vivian Batista da Silva. São Paulo: s.n., 2015.

163 p. ils.; tabs.; anexos; apêndices

Dissertação (Mestrado - Programa de Pós-Graduação em Educação. Área de Concentração: Didática, Teorias de Ensino e Práticas Escolares) -- Faculdade de Educação da Universidade de São Paulo.

1. Diferenças (Educação) 2. Deficiência mental 3. Educação inclusiva 4. Saberes do docente 5. Cultura escolar 6. Patrimônio pedagógico I. Silva, Vivian Batista da, orient. 
Nome: CARVALHO, Cristina Maria Coin de

Título: Os professores frente às diferenças: um estudo de seu patrimônio pedagógico no ciclo II de uma escola pública

Dissertação de Mestrado apresentada ao Programa de Pós-Graduação da Faculdade de Educação da Universidade de São Paulo para obtenção do título de Mestre em Educação.

\section{Banca Examinadora}

Prof. $^{\text {a }}$ Dr. ${ }^{a}$ Vivian Batista Silva - Instituição: FEUSP

Prof. Dr. Marcos Cézar de Freitas - Instituição: UNIFESP

Prof. $^{\text {a }}$ Dr. ${ }^{a}$ Sonia Teresinha de Sousa Penin - Instituição: FEUSP

São Paulo, de de 2015

Aprovado em 
Aos meus pais, Manoel e Marlene, pela confiança desde sempre.

Ao Sérgio, pela presença amorosa e inspiradora.

Aos filhos queridos - Manoela, Ludmila e Vinicius - pela vivacidade e carinho.

Aos irmãos - João Eduardo, Maria Laura, Luciana e Marcos companheiros na vida e na docência. 


\section{Agradecimentos}

À Prof ${ }^{a}$ Dr $^{\mathrm{a}}$ Vivian Batista Silva, por ter acreditado, desde o início, com entusiasmo e força, nesta pesquisa. Agradeço por toda a atenção e delicadeza com que orientou este trabalho e, sobretudo, pela enorme competência com que o fez.

À Prof ${ }^{a}$ Dr $^{a}$ Sônia Teresinha de Sousa Penin e ao Prof. Dr. Marcos Cesar de Freitas, por terem participado da banca de defesa de minha dissertação e pelas preciosas orientações no exame de qualificação.

À Prof. ${ }^{a}$ Dr ${ }^{\mathrm{a}}$ Rita de Cassia Gallego, pela colaboração com o quadro teórico da pesquisa e pelo constante apoio.

À Prof. a Marília Costa Dias, pela semeadura deste trabalho, pelo grande e especial incentivo.

À Luciane Bizari Coin de Carvalho, pelo apoio e incentivos constantes e pela versão do resumo em língua inglesa.

Aos profissionais da escola Vera Cruz - professores, orientadores e coordenadores - que têm, há tanto tempo, me ajudado a ser uma professora pesquisadora.

Aos professores e ao coordenador da escola municipal de ensino fundamental, sujeitos desta pesquisa, profissionais muito engajados na docência, que gentilmente cederam seu tempo e abriram suas salas de aula possibilitando a realização deste trabalho.

A todos meus familiares e amigos, entre os quais muitos professores também, pelo carinho e pelo incentivo.

Aos meus alunos, todos eles, que, nesses mais de vinte anos de docência, têm me ensinado a ser uma professora mais competente. 
Não há possibilidade de pensarmos o amanhã, mais próximo ou mais remoto, sem que nos achemos em processo permanente de 'emersão' do hoje, 'molhados' do tempo que vivemos, tocados por seus desafios.

(Pedagogia da Indignação, 2000, p. 117) 
CARVALHO, Cristina Maria Coin. OS PROFESSORES FRENTE ÀS DIFERENÇAS: Um estudo de seu patrimônio pedagógico no ciclo II de uma escola pública. Dissertação de Mestrado em Educação - Faculdade de Educação - USP - Universidade de São Paulo, São Paulo-SP, 2015.

\section{RESUMO}

A presente pesquisa teve como um dos objetivos contribuir para os estudos sobre a prática dos professores diante do desafio de trabalhar com as diferenças nas salas de aula regulares, tendo como foco o trabalho com alunos com deficiência intelectual e como cenário, a implementação de políticas públicas favoráveis à educação inclusiva. A pesquisa, de caráter qualitativo, além de contar com dados das entrevistas realizadas com professores do ensino fundamental II de uma escola da rede pública do município de São Paulo, recomendada por um CEFAI (Centro de Formação e Acompanhamento à Inclusão), considerou dados coletados na observação da escola. Partiu-se da hipótese de que o professor constrói e reconstrói continuamente um patrimônio pedagógico - conjunto de saberes e representações - ao longo de sua trajetória profissional, utilizando saberes diferenciados e estruturados no interior de uma certa cultura escolar. O objetivo da pesquisa foi identificar e analisar as características do patrimônio pedagógico de um grupo de professores de uma das salas da referida escola, da qual era aluno um menino com deficiência intelectual, e também do coordenador pedagógico responsável pelo ciclo II da escola. A análise de dados levou em conta o conceito de cultura escolar enunciado por Dominique Julia e complementado por Antonio Viñao Frago e também a tipologia dos saberes docentes elaborada por Maurice Tardif e Claude Lessard. O conceito de diferença foi apresentado a partir de reflexões de Goffman (2008), Cury (2002,2005), Silva (2009) e Skliar (2006), que procuram problematizar as relações entre igualdade-diferença/ identidade-diferença. Para analisar as representações referentes à possibilidade de trabalhar com alunos com deficiência intelectual na sala de aula, foi usada a reflexão proposta por Henri Lefebvre, segundo a qual as representações, apesar de serem elaboradas em sociedade, poderiam ser superadas pelos sujeitos. A análise foi organizada em três eixos temáticos relacionados à construção do patrimônio pedagógico: a trajetória pessoal de cada sujeito da pesquisa, os saberes docentes e as representações sobre a possibilidade de se realizar um trabalho pedagógico com alunos com deficiência intelectual na sala regular e as questões institucionais que também incidiriam sobre essa construção. A análise apontou alguns caminhos: a trajetória pessoal, sobretudo o tempo de trabalho na mesma instituição e o envolvimento na construção do seu projeto político pedagógico podem colaborar para uma prática mais eficiente em relação aos alunos com deficiência intelectual; a superação de representações sobre a impossibilidade de um trabalho educacional com esses alunos pode advir do saber experiencial e daquilo que Henri Lefebvre chama de vivido; e finalmente, esta pesquisa apontou para a importância do compartilhamento de patrimônios, que poderia ocorrer nos momentos de reuniões pedagógicas, mas que por questões institucionais nem sempre acontece de forma efetiva e producente.

Palavras-chave: Diferenças (Educação), Deficiência mental, Educação Inclusiva, Saberes do docente, Cultura escolar, Patrimônio Pedagógico 
CARVALHO, Maria Cristina Coin. TEACHERS FACE TO DIFFERENCES: A study of their pedagogical heritage in the elementary public school (Cycle II). Dissertation in Education Faculdade de Educação - USP - Universidade de São Paulo, São Paulo-SP, 2015.

\begin{abstract}
This research aimed to contribute to studies on the practice of teachers facing the challenge of working with differences in regular classrooms, with focus on working with intellectual disability students, and the implementation of public policies for inclusive education as a backdrop. This qualitative research considered the data collected in the observation and from interviews with elementary school (cycle II) teachers of a public school in São Paulo, recommended by a CEFAI (Centre for Training and Monitoring to Inclusion). The hypothesis was that the teacher builds and continuously rebuilds a pedagogical heritage - a set of knowledge and representations - throughout his professional career, using different and structured knowledge within a certain school culture. The objective of the research was to identify and analyze the features of the pedagogical heritage of a group of teachers from one class of the school, in which there were an intellectual disability student, and also the pedagogical coordinator responsible for the elementary school (cycle II). Data analysis took into account the concept of school culture enunciated by Dominique Julia and complemented by Antonio Viñao Frago, and also the typology of knowledge teaching developed by Maurice Tardif and Claude Lessard. The concept of difference was presented from reflections of Goffman (2008), Cury (2002, 2005), Silva (2009), and Skliar (2006), seeking to discuss the relationship between equality-difference/identity-difference. To analyze the representations regarding the possibility of working with students with intellectual disabilities students in the classroom, it was used the reflection proposed by Henri Lefebvre, according to which the representations, despite being prepared in society, could be overcome by the subjects. The analysis was organized into three themes related to the construction of the pedagogical heritage: the personal history of each subject of research, the teaching knowledge and representations about the possibility of developing a pedagogical work with intellectual disability students in regular class, and the institutional issues, that also had been part of this construction. The analysis pointed out some ways: the personal history, specially the working time at the same institution and involvement in shaping their political pedagogical project may contribute to a more efficient practice related to intellectual disabilities students; the overcoming of the representations about the impossibility of an educational work with these students may come from the practical knowledge and from what Henri Lefebvre calls lived; and finally, this research pointed to the importance of sharing heritage, which could occur in pedagogical meetings, but for institutional issues not always happen in an effective and productive manner.
\end{abstract}

Keywords: Differences (Education), Intellectual Disability, Inclusion (Education), Knowledge of teaching, School Culture, Pedagogical heritage 


\section{Lista de figuras}

Figura 1 Página do caderno do aluno J.: exercício de grafia numérica

Figura 2 Página do caderno de J.: exercício de cálculos e de escrita de

números por extenso..

Figura 3 Página do caderno de J.: exercícios com algoritmos. 


\section{Lista de Tabelas}

Tabela 1 Resultados dos últimos Censos Escolares: matrículas de alunos da

Educação Especial e incluídos (elaboração da pesquisadora)

Tabela 2 Dados referentes à trajetória profissional de cada sujeito da

pesquisa 58

Tabela 3 Tipologia dos saberes docentes

Tabela 4 Dados das entrevistas referentes ao saberes da formação profissional. 71

Tabela 5 Dados das entrevistas referentes ao saberes da formação profissional/

representações sobre aluno com deficiência intelectual. 73

Tabela 6 Dados das entrevistas referentes ao saberes das disciplinas 77

Tabela 7 Dados das entrevistas referentes ao saberes da experiência 78

Tabela 8 Dados da pesquisa referentes a suportes estruturais 87

Tabela 9 Empecilhos ao funcionamento das estruturas de apoio 90

Tabela 10 Representações sobre o trabalho em equipe na escola 100 


\section{Lista de Siglas}

AAIDD Associação Americana de Deficiências Intelectual e de Desenvolvimento

AAMR Associação Americana de Retardo Mental

ATE Atendente Escolar

AEE Atendimento Educacional Especializado

CEFAI Centro de Formação e Acompanhamento à Inclusão

CNE/CEB Conselho Nacional de Educação/Câmara da Educação Básica

$\mathrm{CP} \quad$ Coordenador Pedagógico

CIEJA Centro Integrado de Educação de Jovens e Adultos

DUDH Declaração Universal dos Direitos Humanos

DRE Diretoria Regional de Educação

EMEF Escola Municipal de Ensino Fundamental

INEP Instituto Nacional de Estudos e Pesquisas Educacionais Anísio Teixeira

ISEVEC Instituto Superior de Educação Vera Cruz

JBD Jornada Básica Docente

JEIF Jornada Especial Integral de Formação

LDBEN Lei de Diretrizes e Bases da Educação Nacional

MEC Ministério da Educação

PAAI Professor de Apoio e Acompanhamento à Inclusão

PNE Plano Nacional de Educação

PNEEPEI Política Nacional de Educação Especial na Perspectiva da Educação Inclusiva

PPP

Projeto Político Pedagógico 
Sala de Apoio e Acompanhamento à Inclusão

SEESP Secretaria de Educação Especial do Ministério da Educação

SMESP Secretaria Municipal de Educação de São Paulo

TGD Transtornos Globais do Desenvolvimento

UBS Unidade Básica de Saúde

UNESCO Organização das Nações Unidas para a Educação, a Ciência e a Cultura 
1 INTRODUÇÃO

2 OS CAMINHOS DA PESQUISA........................................................................... 50

3 O PATRIMÔNIO PEDAGÓGICO E A TRAJETÓRIA PESSOAL......................... 57

4 O PATRIMÔNIO PEDAGÓGICO: SABERES E REPRESENTAÇÕES .............. 69

5 O PATRIMÔNIO PEDAGÓGICO E A DIMENSÃO

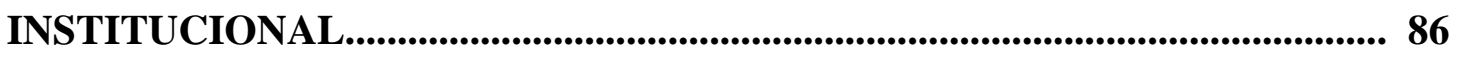

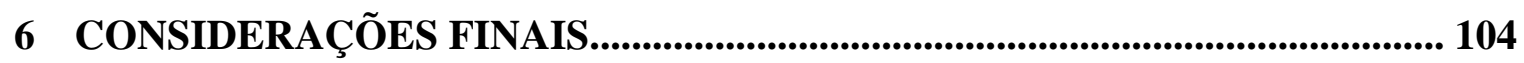

REFERÊNCIAS..................................................................................................108

APÊNDICE A - Dados coletados na $1^{\mathrm{a}}$ etapa da pesquisa................................................... 116

APÊNDICE B - Transcrição das respostas do $2^{\circ}$ e do $3^{\circ}$ grupo de perguntas da entrevista......................................................................................................................... 127

APÊNDICE C- Roteiros das entrevistas.......................................................................... 148

ANEXO 1: DECRETO No 45.415, DE 18 DE OUTUBRO DE 2004.............................. 152

ANEXO 2: PORTARIA N ${ }^{\circ}$ 5.718, DE 17 DE DEZEMBRO DE 2004............................ 155 